\title{
Harmonically resonant cavity as a bunch-length monitor
}

\author{
B. Roberts ${ }^{2, *}$ F. Hannon, ${ }^{1}$ M. M. Ali, ${ }^{3}$ E. Forman, ${ }^{1}$ J. Grames,${ }^{1}$ R. Kazimi, ${ }^{1}$ \\ W. Moore, ${ }^{1}$ M. Pablo, ${ }^{2}$ M. Poelker, ${ }^{1}$ A. Sanchez,${ }^{2}$ and D. Speirs ${ }^{2}$ \\ ${ }^{1}$ Thomas Jefferson National Accelerator Facility, 12000 Jefferson Avenue, \\ Newport News, Virginia 23606, USA \\ ${ }^{2}$ Electrodynamic, 4909 Paseo Del Norte NE suite D, Albuquerque, New Mexico 87113, USA \\ ${ }^{3}$ Department of Physics, Old Dominion University, Norfolk, Virginia 23529, USA
}

(Received 14 December 2015; published 3 May 2016)

\begin{abstract}
A compact, harmonically resonant cavity with fundamental resonant frequency $1497 \mathrm{MHz}$ was used to evaluate the temporal characteristics of electron bunches produced by a $130 \mathrm{kV}$ dc high voltage spin-polarized photoelectron source at the Continuous Electron Beam Accelerator Facility (CEBAF) photoinjector, delivered at 249.5 and $499 \mathrm{MHz}$ repetition rates and ranging in width from 45 to 150 picoseconds (FWHM). A cavity antenna attached directly to a sampling oscilloscope detected the electron bunches as they passed through the cavity bore with a sensitivity of $\sim 1 \mathrm{mV} / \mu \mathrm{A}$. The oscilloscope waveforms are a superposition of the harmonic modes excited by the beam, with each cavity mode representing a term of the Fourier series of the electron bunch train. Relatively straightforward postprocessing of the waveforms provided a near-real time representation of the electron bunches revealing bunch-length and the relative phasing of interleaved beams. The noninvasive measurements from the harmonically resonant cavity were compared to measurements obtained using an invasive RF-deflectorcavity technique and to predictions from particle tracking simulations.
\end{abstract}

DOI: 10.1103/PhysRevAccelBeams.19.052801

\section{INTRODUCTION}

In 2012, we described electron bunch-length measurements made using a prototype harmonically resonant cavity installed on a stand-alone research photoinjector [1]. These measurements indicated that an electron bunch train could excite many modes of the harmonically resonant cavity and the superposition of these modes, as detected by a sampling oscilloscope, resembled electron bunch shapes following relatively simple post-processing. A valid criticism of past measurements was that the research beam line did not possess additional temporal beam monitoring equipment to validate the measurements. In this work, we describe the characterization of an improved harmonically resonant cavity installed at two locations at the Continuous Electron Beam Accelerator Facility (CEBAF) photoinjector. Bunch-length measurements made with the harmonically resonant cavity were compared to measurements using an RF-deflecting cavity technique and to predictions from particle tracking simulations. Good agreement was found between both measurement techniques and model predictions. The harmonically resonant cavity also

\footnotetext{
*Corresponding author. brockr@unm.edu

Published by the American Physical Society under the terms of the Creative Commons Attribution 3.0 License. Further distribution of this work must maintain attribution to the author $(s)$ and the published article's title, journal citation, and DOI.
}

provided useful information about the relative phasing of interleaved pulse trains.

\section{THE HARMONIC CAVITY}

Electron bunches at a pulse repetition rate $w_{0}$ can be described using a Fourier series expansion:

$$
\begin{aligned}
i_{\text {beam }}(t)= & a_{1} \cos \left(w_{o} t+\theta_{1}\right)+a_{2} \cos \left(2 w_{o} t+\theta_{2}\right) \cdots \\
& +a_{n} \cos \left(n w_{o} t+\theta_{n}\right)
\end{aligned}
$$

where $a_{n}$ and $\theta_{n}$ describe the relative amplitudes and phases of each contributing harmonic term. The noninvasive bunch-length monitor cavity was designed to measure each term of the Fourier series expansion:

$$
\begin{aligned}
v_{\text {detected }}(t)= & a_{T M_{010}} \cos \left(w_{0} t+\theta_{010}\right) \\
& +a_{T M_{020}} \cos \left(2 w_{0} t+\theta_{020}\right) \cdots \\
& +a_{T M_{0 n 0}} \cos \left(n w_{0} t+\theta_{0 n 0}\right)
\end{aligned}
$$

where $a_{T M_{0 n 0}}$ and $\theta_{0 n 0}$ describe the relative amplitudes and phases of each detected axially-symmetric transverse magnetic (TM) cavity mode. If the harmonically resonant cavity were perfect, with infinite bandwidth and with all modes perfectly harmonic and equally coupled to the antenna, the amplitude coefficients and phase terms of both equations would be identical (barring a scale factor). However, the cavity and antenna do not have infinite bandwidth and manufacturing imperfections result in some modes being slightly displaced from the intended resonant frequencies. 
Similarly, the cavity antenna does not couple identically to all modes. In sections below, we describe how these imperfections can be corrected during the post-processing of oscilloscope waveforms by multiplying the individual terms of the detected waveform's Fourier series expansion by the cavity's transfer function. In principle, the cavity transfer function can be calculated by dividing Eq. (1) by Eq. (2), but this requires that the electron bunch profile be precisely known. In this work, the cavity transfer function was determined empirically via blind deconvolution [2].

The cavity was designed to resonate at many harmonic $\mathrm{TM}_{0 N 0}$ modes, and to suppress or displace transverse electric (TE) and nonaxially symmetric TM modes within or beyond its operational bandwidth. The shallow saucershaped cavity (Fig. 1) has a mode spectrum free of TE modes for several tens of $\mathrm{GHz}$ because TE modes resonate at frequencies greater than $c / 2 h$ where $c$ is the speed of light and $h$ is the cavity length along the beam's direction of motion. Radial slits cut into the cavity walls do not affect the $\mathrm{TM}_{0 N 0}$ modes which have purely radial wall currents while the $\mathrm{TM}_{\mathrm{MNP}}$ modes with azimuthal mode numbers, $\mathrm{M}$, less than the number of discontinuities are suppressed. Finally, the shape of the cavity was tuned to yield harmonic $\mathrm{TM}_{0 N 0}$ modes. This was accomplished in the design phase by iteratively modifying the cavity geometry and solving for the $\mathrm{TM}_{0 N 0}$ mode frequencies with the field solver POISSON/Superfish [3]. The $\mathrm{TM}_{0 N 0}$ cavity modes are
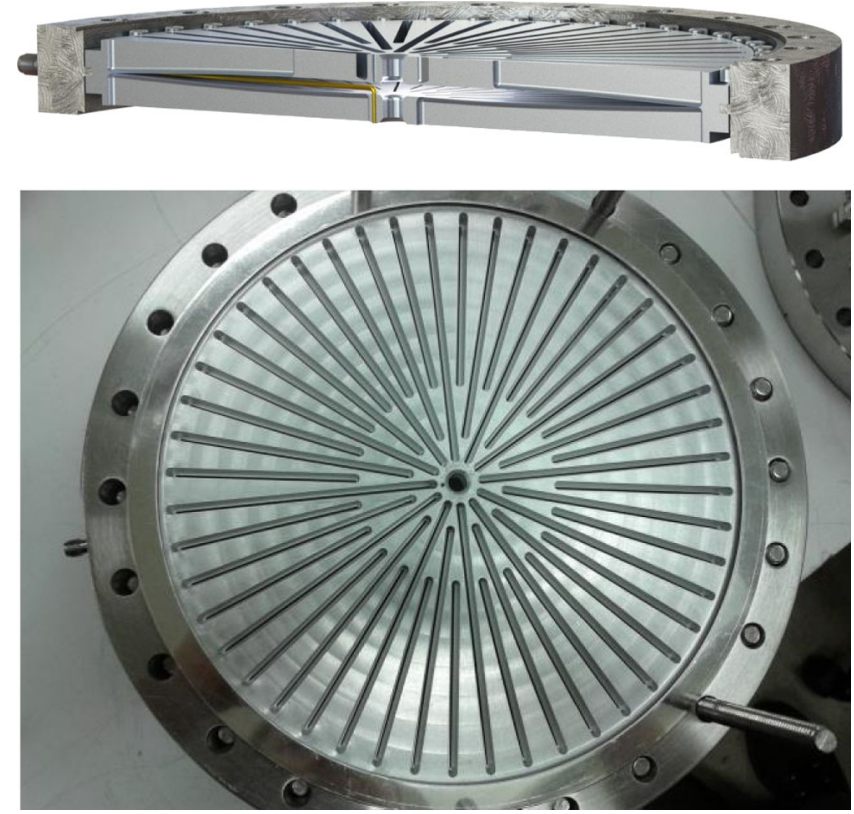

FIG. 1. Top: cut-away view of the harmonically resonant cavity. Bottom: photograph of the cavity nested inside the bore of a 10" double-sided knife edge Conflat flange. Two additional 10" Conflat flanges attach to either side to form the UHV-compatible vacuum vessel. axially symmetric and have a field maximum on the cavity axis, i.e., along the direction of the electron beam motion.

One approach to evaluating temporal characteristics of electron pulse trains at CEBAF would be to use a harmonically resonant cavity with a $499 \mathrm{MHz}$ fundamental resonance frequency, identical to the electron bunch repetition rate produced by three independent photoinjector drive lasers, however, the diameter of such a cavity is large, approximately $60 \mathrm{~cm}$. A cavity this large was deemed too cumbersome for these tests so a harmonically resonant cavity was constructed based on a $1497 \mathrm{MHz}$ fundamental frequency, which is the oscillating frequency of CEBAF accelerating cavities. At $1497 \mathrm{MHz}$, the cavity diameter is just $20 \mathrm{~cm}$, allowing it to fit within a 10" double-sided knife edge Conflat flange. This comparatively compact cavity provided less signal strength compared to one having a fundamental resonant frequency at $499 \mathrm{MHz}$ because some of the harmonic modes at $499 \mathrm{MHz}$ do not excite harmonic modes at $1497 \mathrm{MHz}$. However, the $1497 \mathrm{MHz}$ cavity still provided enough signal to resolve electron bunches at just $1 \mu \mathrm{A}$ average current. Moreover, a compact $1497 \mathrm{MHz}$ cavity was comparatively easier to manufacture, and likely provided more precise control over achieving the required resonance conditions for each higher order mode. The harmonically resonant cavity was also used to evaluate bunches at $249.5 \mathrm{MHz}$ repetition rates. As discussed below, some information is lost when evaluating beams at subharmonic repetition rates of the cavity fundamental frequency, but not information related to bunch shape and duration.

Cavity halves were machined from 6061 aluminum alloy using a vertical computer-numeric-controlled milling machine. The cavity interior surface was cut using a ball-shaped end mill moving along radial toolpaths. Radial slits were first cut into the exterior cavity surface using a wide ball-shaped cutting bit $(3.175 \mathrm{~mm}$ dia.) that did not penetrate the interior surface, and then using a smaller end mill bit $(0.813 \mathrm{~mm}$ dia.) that penetrated the surface creating radial electrical discontinuities along the interior of the cavity. The two halves were bolted together inside a 10" Conflat double-sided knife-edge flange that had been modified to provide a ridge which served to capture the cavity halves. Along the outer periphery of the cavity halves, a $0.813 \mathrm{~mm}$ diameter semicircular groove was cut along the interior surface to accept a soft aluminum wire. Assembling the 48 peripheral bolts compressed the wire and mated the cavity halves electrically. Tightening these bolts deformed the wire and minutely reduced the diameter of the cavity, thereby fine tuning the $\mathrm{TM}_{0 N 0}$ mode spectrum to be integer multiples of CEBAF's $1497 \mathrm{MHz}$ operating frequency. A loop antenna coupler passing between the cavity halves and extending radially from near the cavity aperture (6 $\mathrm{mm}$ dia.) to the vacuum flange was attached to a high quality coaxial SMA vacuum feedthrough. 


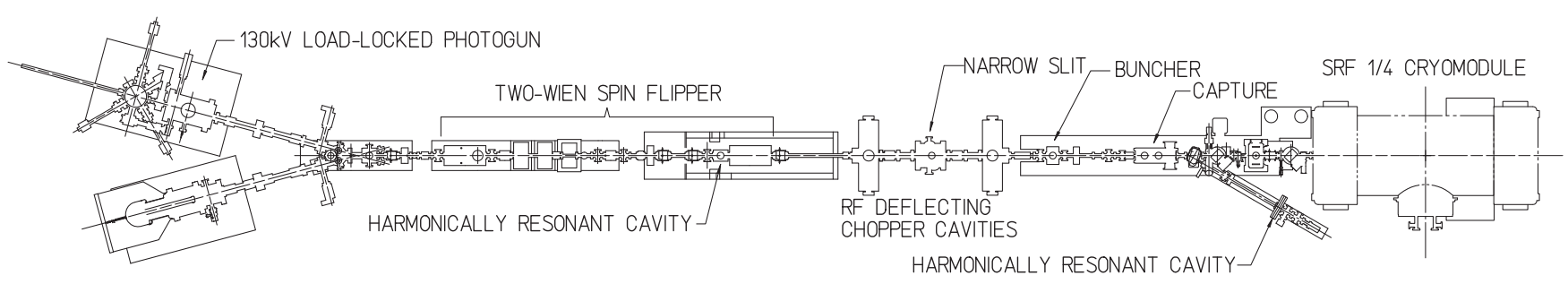

FIG. 2. The CEBAF photoinjector. Note the location of the chopping system, and the two measurement locations of the harmonically resonant cavity bunch-length monitor.

\section{THE EXPERIMENT}

The CEBAF photoinjector [4] is shown in Fig. 2. Pulsedlaser light illuminates a strained-superlattice GaAs/GaAsP photocathode biased at $130 \mathrm{kV}$ inside the high voltage chamber of a load locked photogun [5]. Because the photocathode is thin $(100 \mathrm{~nm})$, the photoemitted electrons form bunches with temporal profiles that resemble, to first order, the laser pulses that produced them. However, as bunches accelerate across the cathode/anode gap, and travel the roughly $14 \mathrm{~m}$ of beamline before reaching the first radio-frequency accelerating cavity, space charge forces serve to alter the temporal distribution of the bunches. The behavior can be dramatic, even at relatively low average current (few $\mu \mathrm{A})$ and bunch charge $(<1 \mathrm{pC})$. The dynamic nature of bunch evolution, and a lack of full understanding of how these dynamics change, for example as the photocathode ages, provided a strong motivation for this work, i.e., the need for a noninvasive bunch-length monitor.

To provide electrons beams to three experimental halls simultaneously, three lasers generate independent $499 \mathrm{MHz}$ pulse trains from a single photocathode inside the photogun [6]. These pulse trains are interleaved in time. Electron beams exit the gun and are bent 15 degrees using an aircore dipole magnet. Two Wien filters and spin rotating solenoids can be used to orient the spin direction at any angle but for this experiment the Wien filters were not energized. Solenoid magnets create beam waists at the 15 degree bend magnet, the first Wien filter, an RF bunching cavity, and at an aperture that was once used to define the transverse emittance of beam produced by a thermionic gun that has since been removed. Downstream of this aperture, two RF-deflector cavities (originally used to produce RF structure on the dc beam produced by the thermionic gun mentioned above) serve to ensure the proper arrival time of electrons at downstream RF components and to "chop off" the head and tail of electron bunches that extend beyond the temporal acceptance of the $130 \mathrm{keV}$ photoinjector.

The chopping system, as it is referred to, uses a pair of $499 \mathrm{MHz} \mathrm{TM}_{210}$ mode cavities [7]. Each cavity is driven in two degenerate orthogonal transverse deflecting modes, phased to sweep beam in a circle with a revolution frequency of $499 \mathrm{MHz}$. Beam at the center of the first cavity is imaged to the center of the second cavity by a pair of counterwound solenoid lenses immediately before and after the chopping apertures, located midway between the two cavities. The amplitudes and phases of the fields in the second cavity are set to completely remove the RF kick from the first cavity. A fully open chopping aperture can transmit a bunch with maximum duration of $111 \mathrm{ps}$.

To validate the noninvasive bunch-length measurements of the harmonically resonant cavity, the first RF-deflector cavity of the chopping system was used as a bunch-length diagnostic. This was accomplished by reducing the width of the chopping aperture to transmit just $18 \mathrm{ps}$ of beam. The temporal profile of electron bunches was mapped by moving bunches across this narrow aperture, by incrementally adjusting the phase of the laser with respect to the deflection cavities while measuring the transmitted current delivered to a downstream Faraday cup.

Under typical CEBAF operating conditions, electron bunches passing beyond the RF deflecting cavities would then be bunched and accelerated to $500 \mathrm{keV}$ using a warm RF capture cavity, and then accelerated to $6.2 \mathrm{MeV}$ using superconducting radio frequency cavities inside a cryostat referred to as the " $1 / 4$ cryomodule".

The harmonically resonant cavity was installed at two locations at the CEBAF photoinjector (see Fig. 2) during two CEBAF scheduled maintenance periods: the first location was upstream of the chopping system, and second location was downstream of the chopping system on a spectrometer beamline beyond a warm-RF capture cavity. Bunch-length measurements made upstream of the chopping system were performed using $130 \mathrm{keV}$ beam, and measurements made downstream were performed using $500 \mathrm{keV}$ beam.

\section{RESULTS}

\section{A. Bunch-length measurements using the RF-deflector technique}

One of the CEBAF drive lasers was used to produce RF-bunched beam at $499 \mathrm{MHz}$, which was then delivered to the RF-deflector cavities. The average beam current, measured using a Faraday cup upstream of components that intercept the beam, was varied by simply adjusting the power of the drive laser light. For each beam current, bunches were moved across the narrow chopping aperture 
by incrementally adjusting the laser phase relative to the RF-deflector cavities in one degree steps. The electron beam passing through the slit was monitored using a downstream Faraday cup, thereby providing a measure of the electron bunch-length and shape. The results presented in Fig. 3a illustrate the extent to which space charge forces influence the electron bunch-length and shape, with bunch-length (full width half maximum, FWHM) increasing from 46 to 132 ps as current increased from 1 to $102 \mu \mathrm{A}$. The laser pulsewidth was measured using a fast photodiode and sampling oscilloscope, and found to be $45+/-2 \mathrm{ps}$, consistent with the electron bunch-length measurement at the lowest current (inset Fig. 3 top). The laser with $499 \mathrm{MHz}$ pulse repetition rate and 45 ps pulsewidth FWHM is referred to as "laser 1".
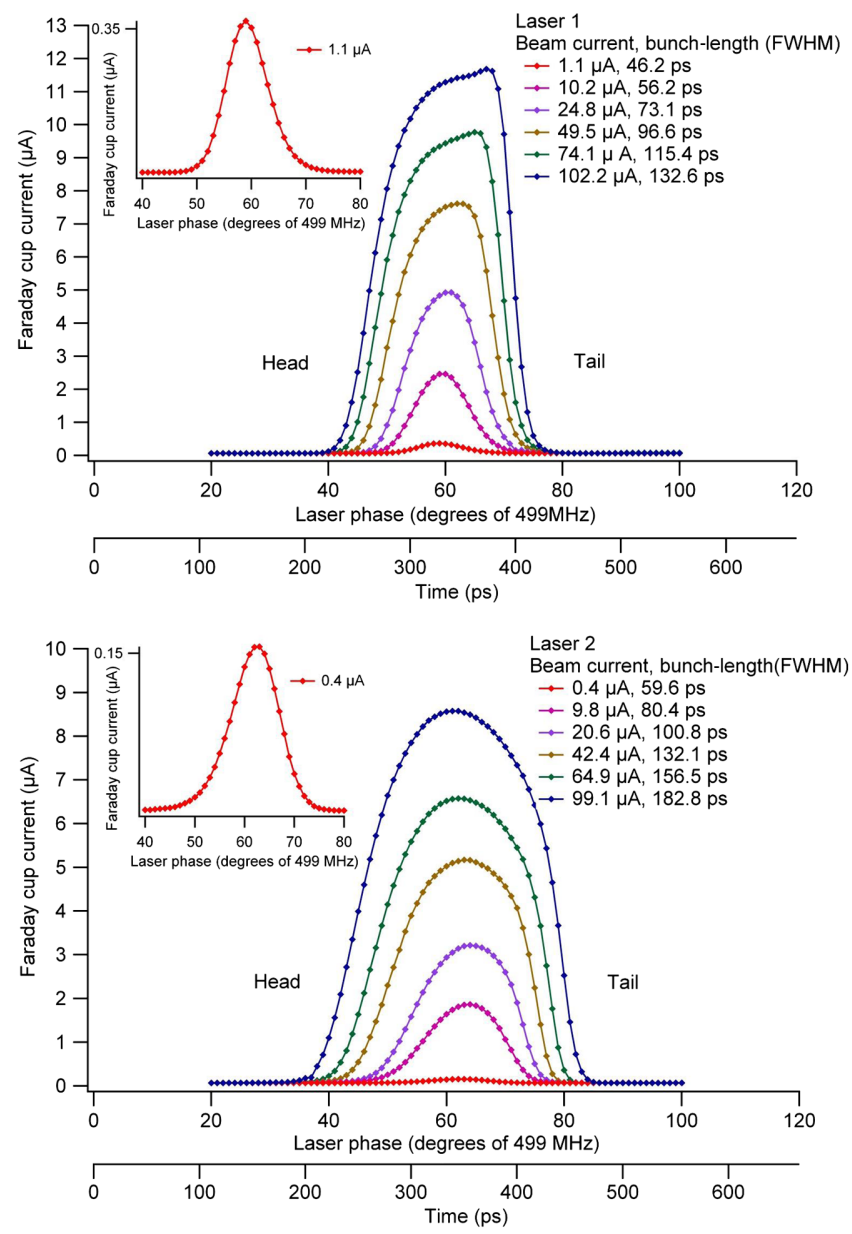

FIG. 3. Electron bunch-length measurements using the RFdeflector cavity, for RF-bunched beam at $499 \mathrm{MHz}$ using "laser 1" with 45 ps optical pulsewidth FWHM (top) and $249.5 \mathrm{MHz}$ using "laser 2" with 60 ps optical pulsewidth FWHM (bottom), for average beam current ranging from 1 to $100 \mu \mathrm{A}$. The insets provide more detail for the lowest current measurements which suffer the least amount of space charge induced bunch-length growth, and therefore most closely resemble the laser optical pulse shape.
Similar measurements were repeated using another drive laser, referred to as "laser 2", operating at $249.5 \mathrm{MHz}$ and with a longer optical pulsewidth, $60+/-2$ ps (Fig 3b). The same current range was explored but resulting in twice the bunch charge as the previous set of measurements. Measured bunch-length increased more dramatically, from 62 to 184 ps FWHM, as current increased from 0.4 to $100 \mu \mathrm{A}$. The bunch-length measurement at the lowest current was consistent with the laser optical pulsewidth measurement (inset Fig. 3 bottom). Note that at higher currents, for both repetition rates, the electron bunch shape became asymmetric. This behavior is routinely observed at CEBAF, and consistent with the first report of synchronous photoinjection [8].

To calculate the bunch-length values included in the legend of Fig. 3, the width of the chopper slit was deconvolved from raw data. This was accomplished using two closely agreeing approaches; numerically using the software program IGOR [9], and the simple calculation strictly applicable for a symmetric Gaussian temporal beam profile and a rectangular window function, $t=\sqrt{t_{\text {meas }}^{2}-18^{2}}$, where $t_{\text {meas }}$ describes the width of raw-data trace (FWHM) and 18 ps represents the width of the narrow chopper slit. To assign errors to the results, the width of the narrow slit was assumed to possess some uncertainty, $+/-1 \mathrm{ps}$, that would result due to improper chopper setup, for example by not operating the RF-deflector cavities or nearby solenoid magnets at design values. But because the electron bunches are relatively long compared to the size of the narrow slit, an uncertainty in slit width of this scale has very little impact on the end result. Bunch-length values stated in the legends of Fig. 3 are assumed accurate to $\sim 2 \%$.

\section{B. Bunch-length measurements using the harmonically resonant cavity}

After making the invasive bunch-length measurements using the RF-deflecting cavity, noninvasive measurements were performed using the harmonically resonant cavity at the location upstream of the RF-deflector cavity. A Tektronix model SD-30 oscilloscope sampling head with $40 \mathrm{GHz}$ bandwidth was attached directly to the smavacuum feedthrough of the harmonically resonant cavity. The sampling head was connected to a Tektronix Model 11801B digital sampling oscilloscope using an extender cable. Figure 4a shows a representative oscilloscope trace from the harmonically resonant cavity for beam at $10 \mu \mathrm{A}$ (red) and the imagined true shape of the electron bunches passing through it (blue). The "distortions" seen in the detected waveform stem from small imperfections in the cavity geometry, and as a result of imperfect antenna coupling to all cavity modes. Slightly off-resonant cavity modes, combined with nonuniform antenna coupling, result in phase and amplitude differences between the 

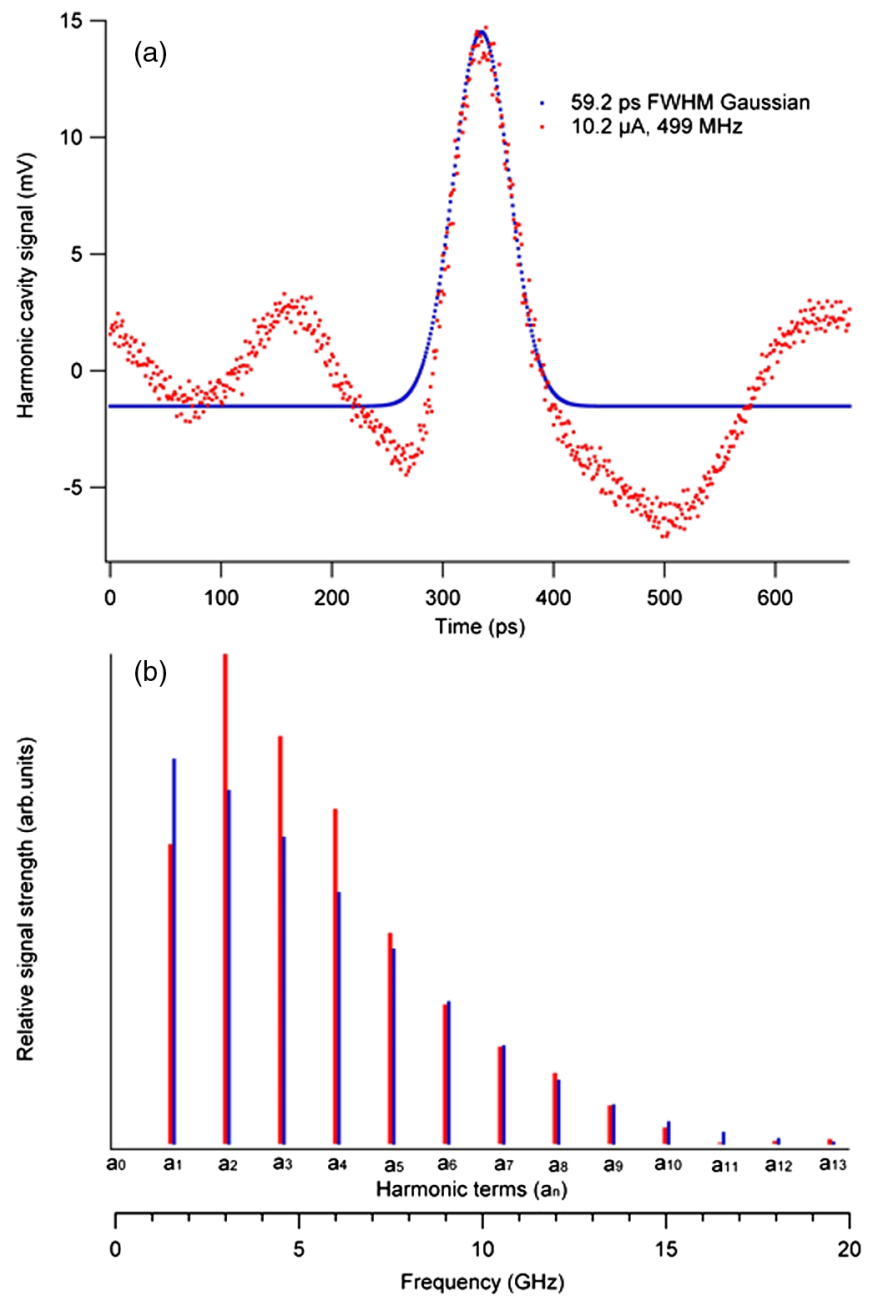

FIG. 4. (a) the raw oscilloscope trace for $10 \mu \mathrm{A}$ beam through the harmonically resonant cavity at $499 \mathrm{MHz}$ repetition rate (red) and the assumed bunch profile that provided a transfer function that produced transformed oscilloscope waveforms with the minimum signal outside the central bunch (blue), (b) the relative signal strength for each resonant mode of the harmonically resonant cavity: red, cavity as measured, and blue, the response of an ideal harmonic cavity.

measured response of each cavity mode and ideal modes representative of the beam's Fourier series (Fig. 4b).

If the resonant frequency of an individual cavity mode is slightly off design, the beam can still excite this mode provided the beam's Fourier term is not outside the mode's resonance curve. But driving a cavity mode off the resonance peak causes a decrease in detected amplitude, and it introduces a phase shift between the beam and the excited mode. The error associated with this mode can be corrected using a single complex multiplier that "unshifts" the phase offset and scales the detected amplitude. A series of complex multipliers - one for each cavity mode — can be created. This series expansion has a functional form similar to Eq. (1) and it is called the cavity transfer function. The beam's true Fourier series representation can be obtained by multiplying each term of the Fourier series of the detected signal by each term of the cavity transfer function. The challenge associated with this approach relates to the fact that the cavity transfer function is not explicitly known, rather it must be deduced in a sensible manner. However, once the transfer function is known, it can then be used to correct the cavity systematic errors for all subsequent data, independent of new bunch shapes.

To generate a sensible cavity transfer function, the method of blind deconvolution [2] was employed. A low-current waveform obtained using "laser 1" operating at $499 \mathrm{MHz}$ and with 45 ps optical pulsewidth was selected because space charge forces were less likely to influence the shape of the bunch. Fourier series were created for "guessed" Gaussian bunch-lengths ranging from 40 to $60 \mathrm{ps}$, in $2 \mathrm{ps}$ increments. Candidate transfer functions were then calculated by dividing the Fourier series of the guessed profiles by the Fourier series of the actual measured waveform. Each of these candidate transfer functions was then used to correct the waveforms of
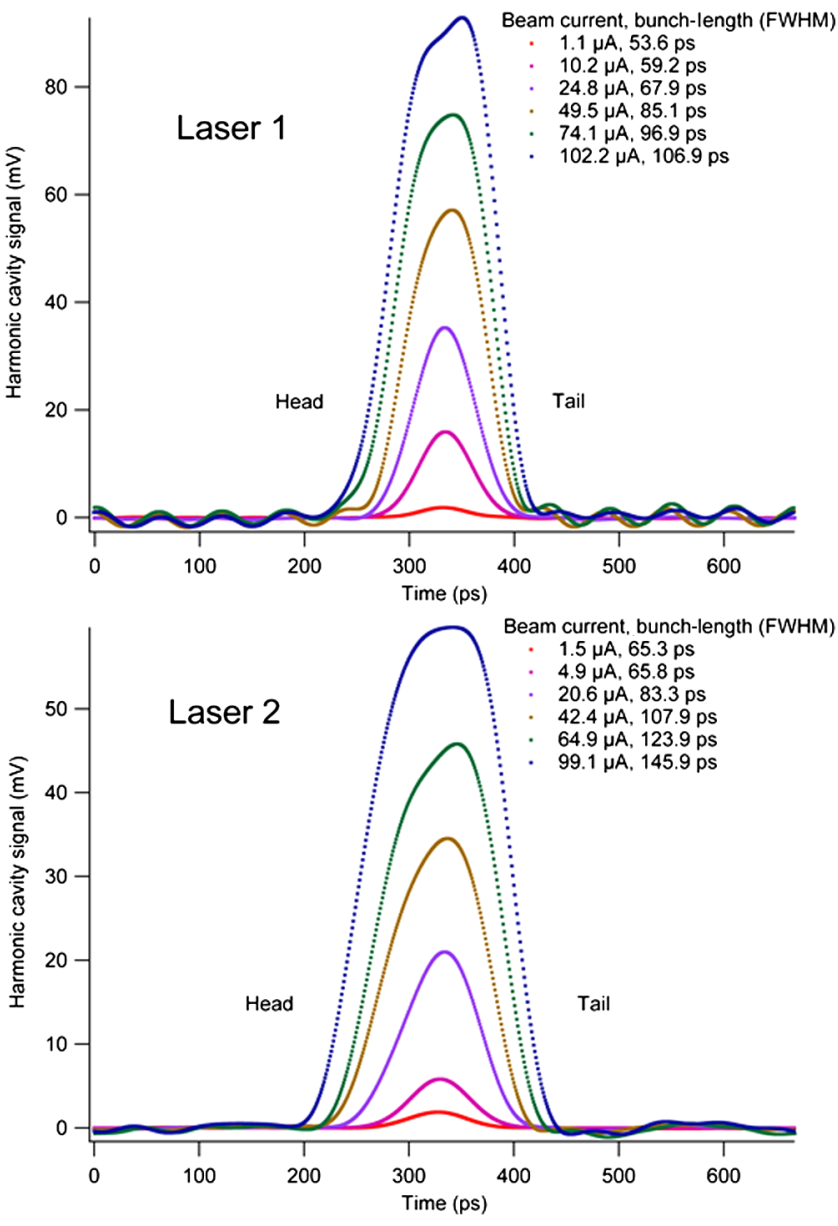

FIG. 5. Bunch-length plots using the harmonically resonant cavity for electron beams at $499 \mathrm{MHz}$ using "laser 1" with $45 \mathrm{ps}$ optical pulsewidth FWHM (top) and 249.5 MHz using "laser 2" with 60 ps optical pulsewidth FWHM (bottom), for different extracted beam currents. These plots were obtained by transforming the raw oscilloscope waveforms. 
measurements of longer bunches arriving at the harmonically resonant cavity. Most of these transfer functions did a poor job of correcting waveform distortions, particularly at higher currents. The transfer function deemed most accurate was one that generated the least amount of signal outside the central bunch. It produced the corrected waveforms shown in Fig. 5a, where distortions and oscillations outside the central bunch were effectively minimized. The same methodology was employed for measurements obtained using "laser 2" (Fig. 5b). Specific post-processing steps are outlined in the Appendix. Electron bunch-length increased at higher beam currents, just as observed for bunch-length measurements made using the RF-deflector technique. Unsurprisingly, over the same current range 1-100 $\mu \mathrm{A}$, space charge forces had more impact on beam produced at $249.5 \mathrm{MHz}$ compared to $499 \mathrm{MHz}$. The bunchlength at $249.5 \mathrm{MHz}$ increased from 62 to $147 \mathrm{ps}$ compared to beam produced at $499 \mathrm{MHz}$ which increased from 63 to 104 ps. Notice that at higher beam current, the harmonically resonant cavity reported slightly asymmetric beam profiles, similar to those observed using the RF-deflector measurement technique but with less detail, particularly when comparing traces obtained at $499 \mathrm{MHz}$.

During the 2012 experiments described in Ref. [1] using a $1497 \mathrm{MHz}$ harmonically resonant cavity, it was observed that oscilloscope waveforms were similar when the drive laser repetition rate was set to 1497 or $499 \mathrm{MHz}$. On one hand, this made sense because for both repetition rates, the cavity was being excited by similar bunch shapes. But at $499 \mathrm{MHz}$, it was puzzling that the harmonically resonant cavity could provide sufficient fidelity when detecting only one third of the beam's Fourier series harmonic content. We investigated this phenomenon graphically by creating hypothetical $499 \mathrm{MHz}$ bunch shapes, calculating their
Fourier series representations, and then calculating their inverse transforms using only terms harmonic at $1497 \mathrm{MHz}$. As expected, information was lost by discarding harmonic terms in the original beam's Fourier series. Specifically, the exact location of the bunch within the 2004 ps period of the $499 \mathrm{MHz}$ cycle was unknown. However, when the electron bunch-length was less than the period of the $1497 \mathrm{MHz}$ fundamental frequency, the shape and duration of electron bunches produced at subharmonics of $1497 \mathrm{MHz}$ were measured without loss of fidelity.

\section{Particle tracking simulations}

The bunch-length values measured using the harmonically resonant cavity and via the RF-deflecting cavity method are not expected to be identical, because the two devices were separated by 2.1 meters and electron bunches continue to grow while traveling this extra distance. To tie together the measurements of both devices, the modeling program ASTRA [10] was used to predict the electron bunch-length at both locations. Agreement between ASTRA predictions and the bunch-length measurements using the RF-deflector cavities was deemed essential, to validate the ASTRA predictions of bunch-length measurements at the location of the harmonically resonant cavity.

ASTRA tracks macroparticles through user-defined external fields while including the effects of space charge forces on the particle cloud. Three dimensional field maps of complex components (i.e., asymmetric) can be used, but for simple, cylindrical elements such as solenoids used at the CEBAF photoinjector, basic on-axis field maps provide a good approximation with reduced computation time. Calculations show dipole magnets at the CEBAF injector contribute less than $0.01 \%$ increase in the bunch-length,
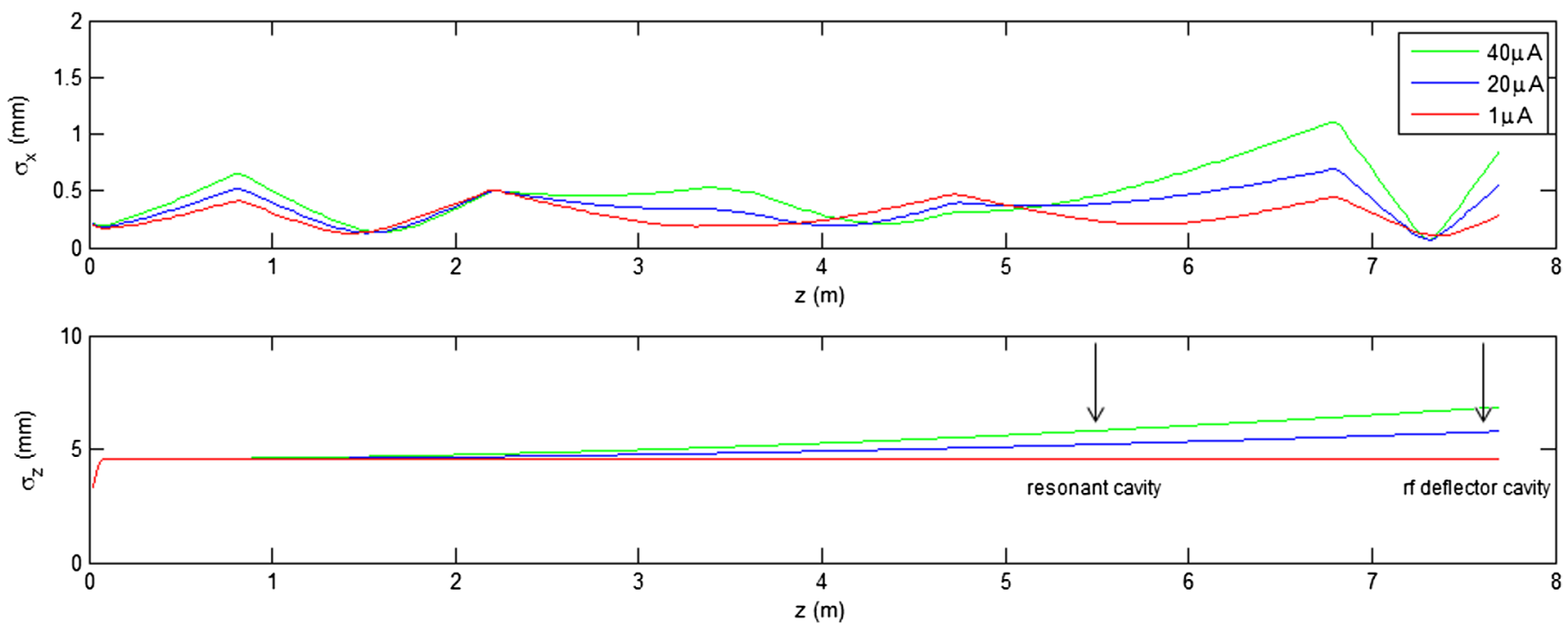

FIG. 6. Simulation results showing the transverse (top) and longitudinal (bottom) rms sizes the beam as a function of distance from the photocathode for various beam currents using the laser at $249.5 \mathrm{MHz}$. The arrows denote the locations of the harmonically resonant cavity and the narrow aperture between the two RF-deflecting cavities. 
due to the beam's very low energy spread, and because the R56 of a short bend magnet is small compared to velocity effects. For this reason bending magnets (dipoles and steering magnets) were ignored in the simulation. The $\mathrm{RF}$ deflecting cavities were also ignored because the impact of these elements effectively "cancel out" when beam is returned on axis.

The particle distribution at the photocathode was defined using laser spatial profile measurements obtained with a CCD, or charge coupled device, camera. Both lasers had Gaussian transverse spatial profiles, $0.25 \mathrm{~mm}$
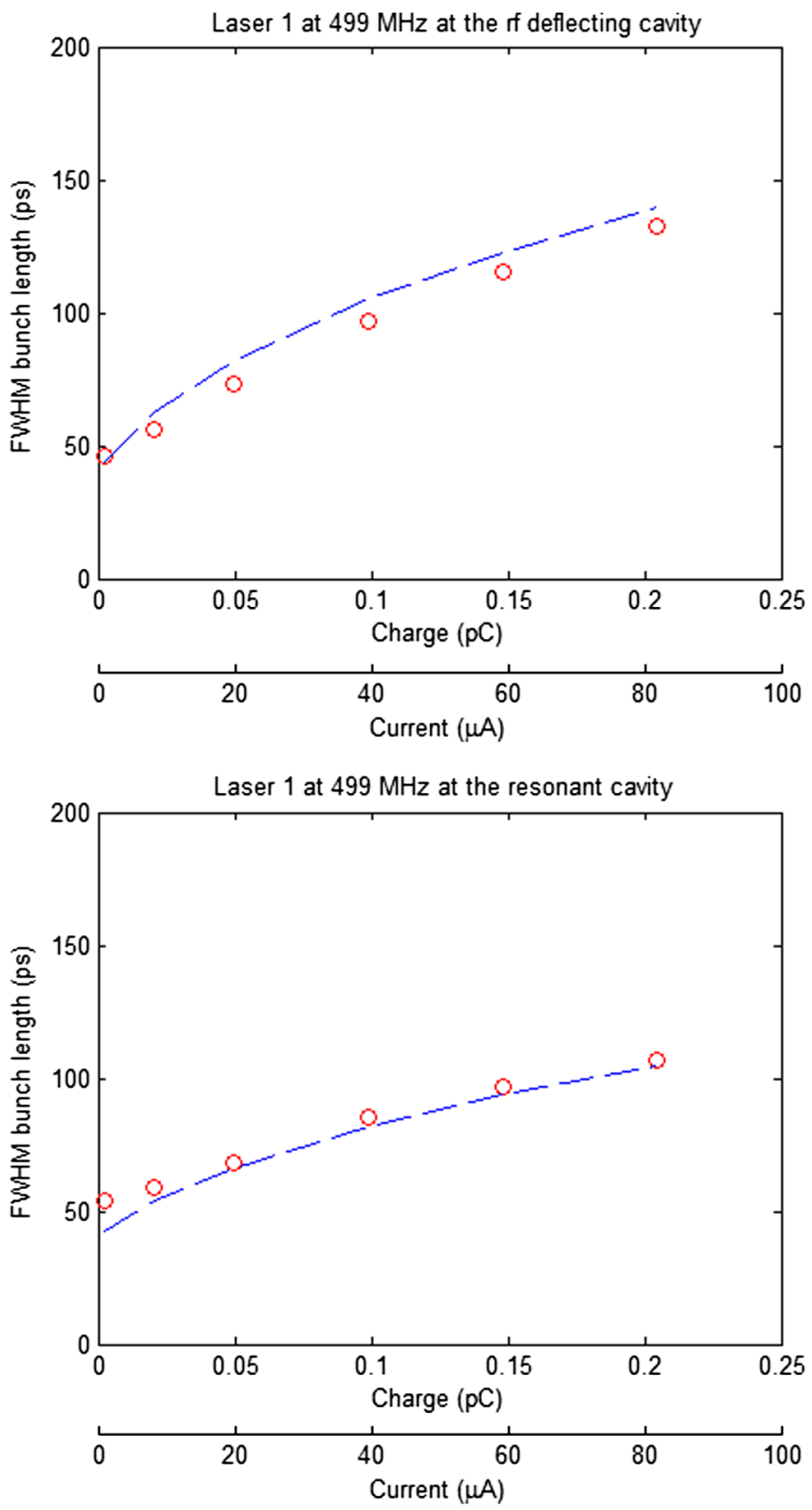

rms. As previously noted, laser pulse widths were $45 \mathrm{ps}+/-2 \mathrm{ps}$ (FWHM) for "laser 1" operating at $499 \mathrm{MHz}$ laser and $60 \mathrm{ps}+/-2 \mathrm{ps}$ (FWHM) for "laser 2 " at $249.5 \mathrm{MHz}$. But because of the limited bandwidth of the fast photodiode used to evaluate the laser temporal characteristics, simulations were repeated using a superGaussian shape of increasing order to represent a temporal profile that would become increasingly uniform. The best fit to the measurements was obtained using a simple Gaussian shape. The photocathode was assumed to be a prompt emitter because it was very thin and the
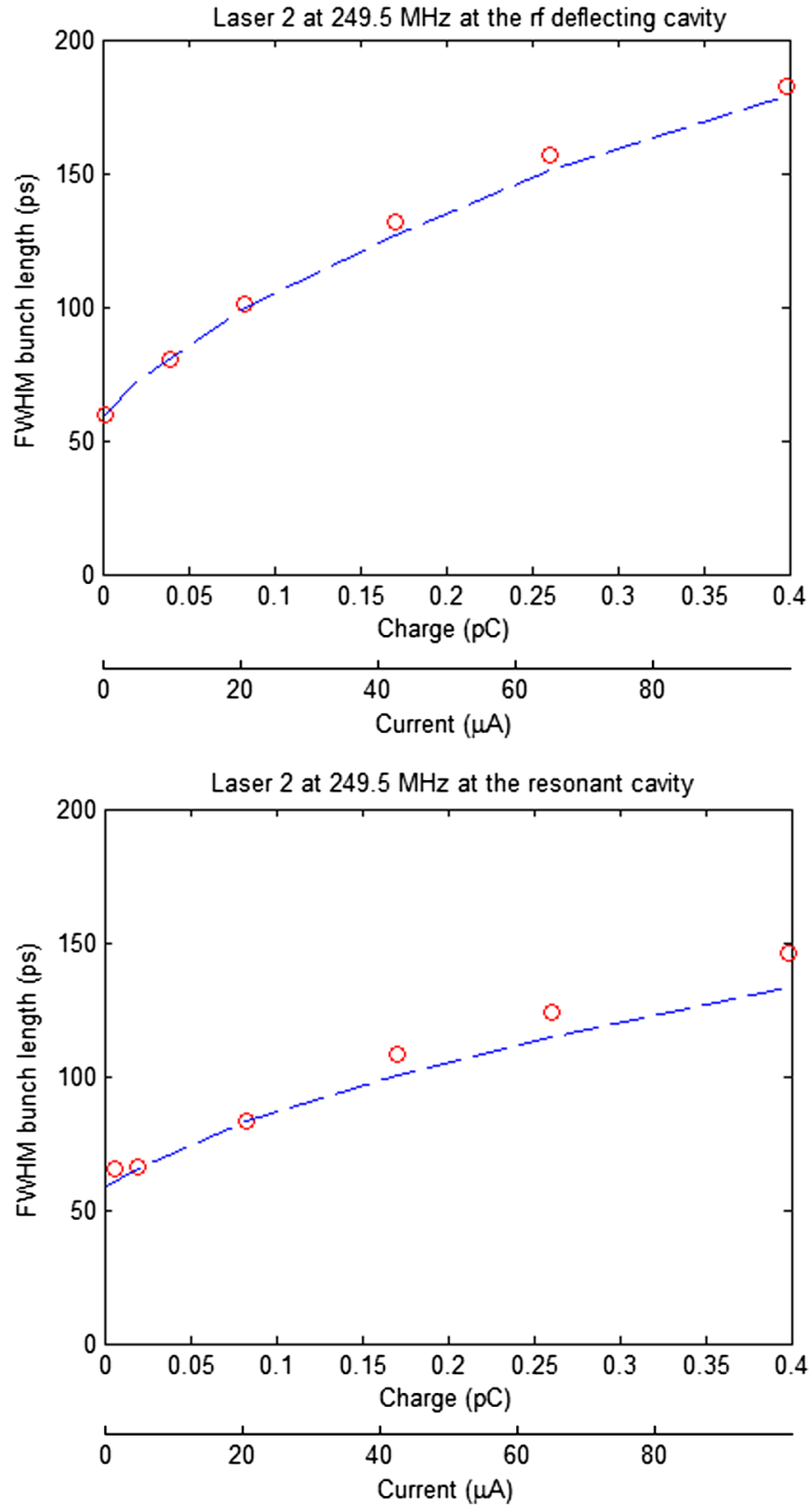

FIG. 7. Electron bunch-length as a function of bunch charge (average current varied from 1 to $100 \mu \mathrm{A}$ ) with measurements obtained using the invasive RF-deflecting cavity technique (top) and the harmonically resonant cavity (bottom), together with simulation results using the particle tracking code ASTRA. Left) measurements made using "laser 1" at $499 \mathrm{MHz}$ and, Right) measurements made using "laser 2" at $249.5 \mathrm{MHz}$. 
thermal energy properties of the photocathode used in the simulations were those discussed in Ref. [11]. The results presented are based on simulations made with 10,000 macroparticles. Figure 6 shows examples of the beam evolution-transverse size $\sigma_{x}$, and bunch-length $\sigma_{z}$-for beam originating at the photocathode and extending through the injector, for several beam currents at a bunch repetition rate $249.5 \mathrm{MHz}$.

Particle distributions were obtained at the harmonically resonant cavity and at the narrow aperture between the two rf-deflecting cavities. To obtain corresponding FWHM values, histograms of the longitudinal position of particles were interpolated using a cubic spline function. Compiled bunch-length values obtained using both measurement techniques are presented in Fig. 7 together with simulation results using both laser repetition rates, 249.5 and $499 \mathrm{MHz}$. There is clearly very good agreement between the simulation and the measurements made using the RF-deflector technique. There is also good agreement between simulation and measurements made using the harmonically resonant cavity, although two features warrant discussion. For "laser 2" operating at $249.5 \mathrm{MHz}$, the harmonically resonant cavity over-estimates bunch-length at higher currents by $\sim 10 \%$. This could be an indication that the empirically determined transfer function needs further refinement. Second, the measurements made using "laser 1" at $499 \mathrm{MHz}$ overestimate the bunch-length at low current, reporting a $54 \mathrm{ps}$ bunch-length at $1 \mu \mathrm{A}$ rather than the expected value of $45 \mathrm{ps}$ representative of the laser pulse that produced the beam. This discrepancy illustrates the resolution limit of the harmonically resonant cavity, at least at low current.

It is worth mentioning that ASTRA simulations did not predict asymmetric electron bunch shapes at higher current and bunch charge, whereas both measurement techniques indicated asymmetric bunch profiles.

\section{Phase detection and interleaved pulse trains}

The multiuser nature of CEBAF requires independently controlled beams interleaved in time and with a precise phase/time interval between bunch trains. This feature of CEBAF provided an excellent opportunity to evaluate the phase detecting capability of the harmonically resonant cavity. For these measurements, a splitter was attached to the cavity antenna, delivering approximately half of the generated signal to the sampling oscilloscope and the other half to a zero-bias Schottky diode rectifier. The output of the Schottky diode was delivered to a digital voltmeter. With this new configuration, independently controlled beams were generated using two drive lasers at $249.5 \mathrm{MHz}$, and delivered to the harmonically resonant cavity. The arrival time of one beam was held constant, while the arrival time of the other beam was varied to effectively pass the electron bunches of one beam across

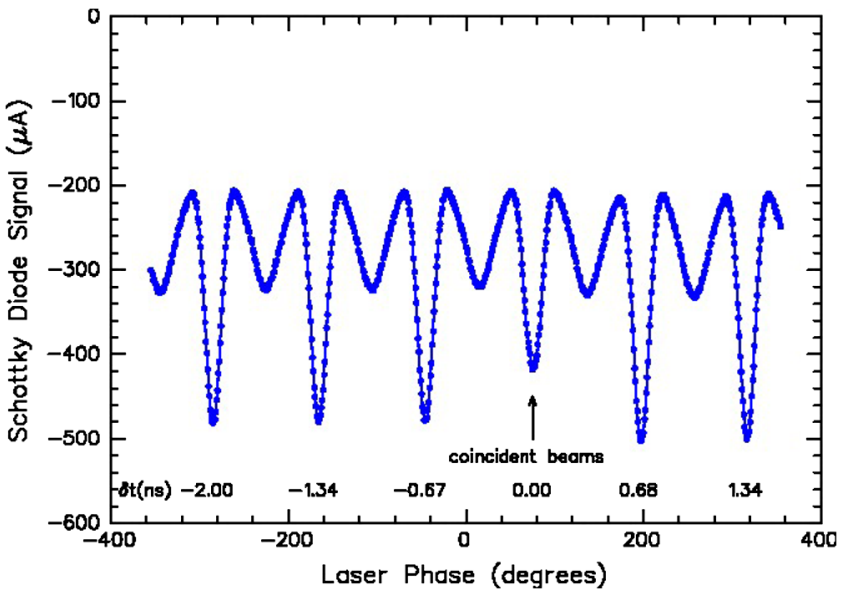

FIG. 8. Current from the zero-bias Schottky diode as one electron bunch train was passed across the other by varying the RF-phase of one drive laser. Both beams were produced using lasers with $249.5 \mathrm{MHz}$ pulse repetition rate. The prominent dips corresponds to precise time intervals between bunches, $n \times 668$ ps. The dip with smallest magnitude corresponds to bunches coincident in time.

the other. Each prominent dip visible in the Schottky diode signal shown in Fig. 8 corresponds to a precise time interval between bunches, $n / 1497 \mathrm{MHz}$, or $n \times 668$ ps. The magnitude of these dips relates to the effectiveness of each condition to excite the modes of the cavity. When two bunches are separated by $1 / 1497 \mathrm{MHz}$, or $668 \mathrm{ps}$, the dominant modes of the cavity are excited yielding the largest Schottky diode signal. The smallest signal corresponds to two bunches coincident in time, a situation indistinguishable from one beam at $249.5 \mathrm{MHz}$. The capability of the harmonically resonant cavity to identify precise timing relationships between independent pulse trains is a very useful feature for CEBAF machinesetup, providing a quick and accurate means to set the laser phases of the three drive lasers.

\section{E. Measurements at $500 \mathrm{keV}$}

Measurements were also made with the harmonically resonant cavity placed downstream of the "capture" section (Fig. 2), with beam at $500 \mathrm{keV}$. Figure 9 (top) shows a raw oscilloscope trace obtained for $499 \mathrm{MHz}$ beam passing through the harmonically resonant cavity at $25 \mu \mathrm{A}$ average current, with a prominent peak $35 \mathrm{ps}$ wide (FWHM). As the beam current was adjusted, the magnitude of the peak-to-peak signal scaled with current, but the temporal width of the central peak remained the same. Particle tracking simulations suggest the actual bunch-length at this location to be much shorter, closer to $\sim 1$ ps. Therefore, measurements at this location represent an assessment of the resolution bandwidth of the device. These measurements indicate that the cavity can resolves bunches as short as 235 ps FWHM, consistent with the spectral content of measured waveform's Fourier series 


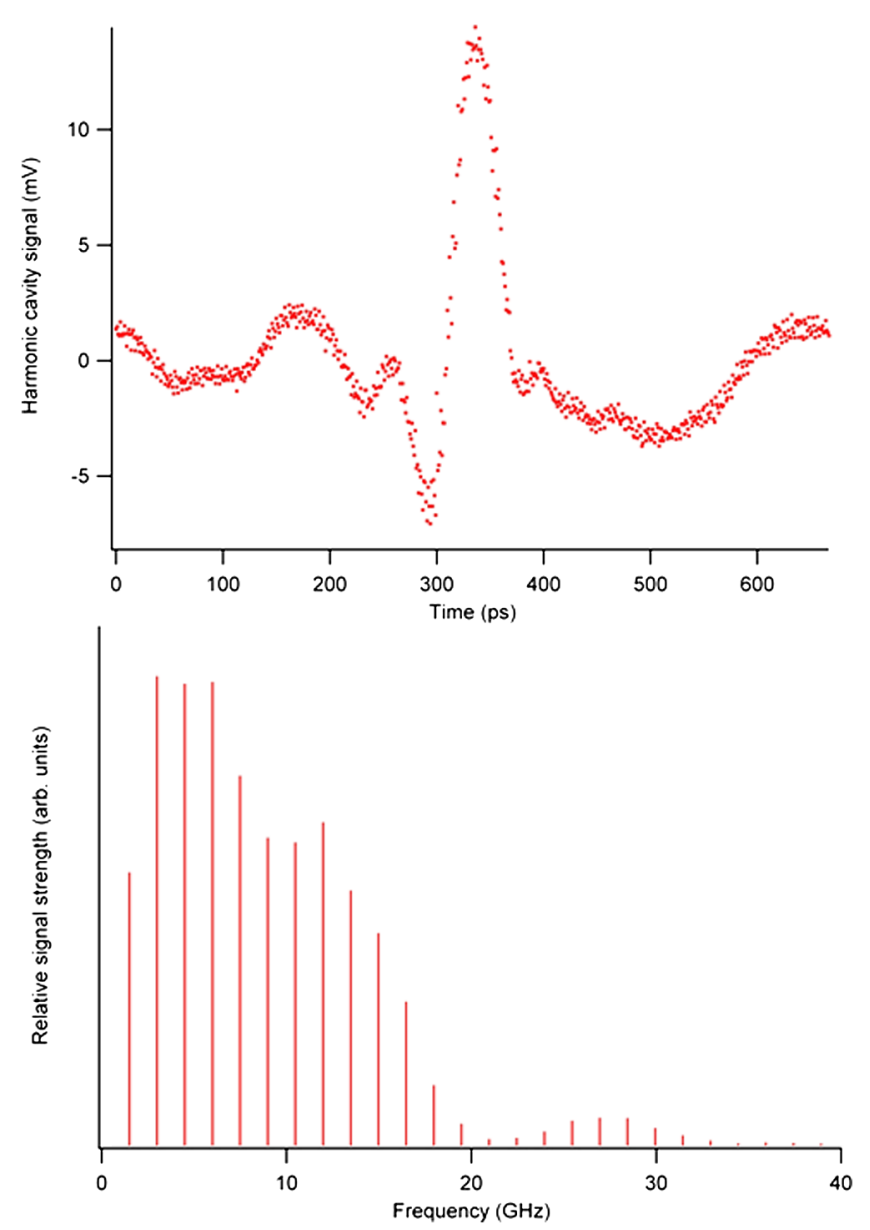

FIG. 9. Top: Raw oscilloscope waveform showing a bandwidth-limited electron bunch-length measurement for $25 \mu \mathrm{A}$ average current at $499 \mathrm{MHz}$ repetition rate and $500 \mathrm{keV}$ beam energy. Bottom: the spectral content of the same waveform indicating the harmonically resonant cavity's operational bandwidth of $\sim 20 \mathrm{GHz}$.

spectral content which extended to $20 \mathrm{GHz}$ (Fig. 9 bottom).

\section{CONCLUSION}

A novel noninvasive bunch-length measurement technique was validated against a traditional invasive RF-deflector cavity technique, and using particle tracking simulations. The compact harmonically resonant cavity allows near-real time evaluation of electron bunches as short as $35 \mathrm{ps}$. In the future, effort will be devoted to developing a computer algorithm to more accurately determine the harmonically resonant cavity transfer function. It was shown that the cavity also provides very practical information on the relative phasing of interleaved pulse trains, a feature that will reduce the setup time of the CEBAF photoinjector. It is possible this feature could be exploited at other locations at CEBAF, for example, where beams at higher energy are combined for recirculation through the linac.

\section{ACKNOWLEDGMENTS}

Authored by Jefferson Science Associates under U.S. DOE Contract No. DE-AC05-84ER40150. Electrodynamic is funded by the DOE's SBIR program DE-SC0009509.

\section{APPENDIX: CAVITY TRANSFER FUNCTIONS}

The post-processing steps to transform raw oscilloscope waveforms into bunch-length measurements using a harmonically resonant cavity with $1497 \mathrm{MHz}$ fundamental frequency:

TABLE I. The transfer functions for the harmonically resonant cavity installed at the CEBAF photoinjector for different drive laser conditions.

\begin{tabular}{cccc}
\hline \hline Real & Imaginary & Magnitude & Phase (Degrees) \\
\hline Laser 1, 499 MHz & & & \\
1 & 0 & 1 & 0 \\
0.68 & 1.09 & 1.28 & 58 \\
0.72 & 0.01 & 0.72 & 1.06 \\
0.54 & -0.53 & 0.75 & -44.44 \\
0.75 & 0.05 & 0.75 & 3.61 \\
0.92 & 0.12 & 0.93 & 7.46 \\
1.02 & -0.05 & 1.02 & -3.06 \\
1.01 & 0.03 & 1.01 & 1.70 \\
0.88 & 0.22 & 0.91 & 14.24 \\
0.77 & 0.67 & 1.02 & 41.2 \\
0.92 & 1 & 1.36 & 47.41 \\
0.95 & 5.94 & 6.02 & 80.96 \\
-1.67 & 0.54 & 1.75 & 162.14 \\
-0.03 & -0.59 & 0.59 & -93.23 \\
0.08 & -0.37 & 0.38 & -78.47 \\
-0.08 & -0.13 & 0.15 & -120.27 \\
-0.01 & -0.05 & 0.05 & -103.62 \\
0 & 0 & 0 & 0 \\
Zeros to $N / 2$ & 0 & 0 & 0 \\
\hline \hline
\end{tabular}

\begin{tabular}{lccc}
\hline \hline Laser 2, 249.5 MHz & & & \\
1 & 0 & 1 & 0 \\
0.52 & 0.86 & 1 & 58.74 \\
0.69 & 0.13 & 0.7 & 10.8 \\
0.68 & -0.47 & 0.83 & -34.9 \\
0.74 & 0.09 & 0.74 & 6.67 \\
1.03 & 0.12 & 1.03 & 6.41 \\
1.29 & -0.23 & 1.31 & -10.28 \\
1.32 & -0.25 & 1.34 & -10.88 \\
1.19 & -0.11 & 1.19 & -5.32 \\
1.08 & 0.31 & 1.13 & 16.21 \\
0.83 & 0.28 & 0.87 & 18.71 \\
0.29 & 0.18 & 0.34 & 32.36 \\
0.41 & 0.23 & 0.47 & 29.63 \\
-0.41 & 0.11 & 0.42 & 164.84 \\
0.16 & 0.07 & 0.17 & 25.53 \\
0.03 & 0.03 & 0.05 & 40.66 \\
0 & 0 & 0 & 0 \\
0 & 0 & 0 & 0 \\
Zeros to $N / 2$ & 0 & 0 & 0 \\
\hline \hline
\end{tabular}


1. Acquire the oscilloscope waveform with at least 668 ps of information, corresponding to one complete wavelength at $1497 \mathrm{MHz}$.

2. Trim the acquired waveform to be to as close as possible to $668 \mathrm{ps}$. The number of points in this trace will be referred to as $\mathrm{N}$ in the following steps.

3. Calculate the waveform's complex discrete Fourier transform. Programs such as IGOR and Python produce $\mathrm{N} / 2$ positive frequency terms in real + imaginary format. (Python produces negative frequency terms as well as positive ones due to the mirror symmetry of the Fourier transform about the $\mathrm{Y}$ axis, these can be discarded)

4. Calculate the product of the returned FFT and the cavity's transfer function. The transfer function is also two columns of numbers in real + imaginary format. The transfer functions used to transform the data in Fig. 5 is provided in Table 1. The two series of complex numbers should be line-by-line multiplied, following the rules of complex multiplication, amplitudes are multiplied and phases added.

5. Calculate the inverse Fourier transform of the product to produce a distortion corrected bunchshape.

[1] B. Roberts, R. R. Mammei, M. Poelker, and J. L. McCarter, Compact non-invasive electron bunch-length monitor, Phys. Rev. Accel. Beams 15, 122802 (2012).

[2] M. Cannon, Blind deconvolution of spatially invariant image blurs with phase, IEEE Trans. Acoust. Speech Signal Process. 24, 58 (1976); E. Y. Lam and J. W. Goodman, Iterative statistical approach to blind image deconvolution, J. Opt. Soc. Am. A 17, 1177 (2000).
[3] K. Halbach and R. F. Holsinger, SUPERFISH - A computer program for evaluation of RF cavities with cylindrical symmetry, Particle Accelerators 7, 213 (1976).

[4] C. K. Sinclair, M. Poelker, P. A. Adderley, B. M. Dunham, J. C. Hansknecht, P. Hartmann, J. S. Price, P. M. Rutt, W. J. Schneider, and M. Steigerwald, A high average current polarized electron source with long cathode operational lifetime, Phys. Rev. Accel. Beams 10, 023501 (2007).

[5] P. A. Adderley, J. Clark, J. Grames, J. Hansknecht, K. Surles-Law, D. Machie, M. Poelker, M. L. Stutzman, and R. Suleiman, Load-locked dc high voltage GaAs photogun with an inverted-geometry ceramic insulator, Phys. Rev. Accel. Beams 13, 010101 (2010).

[6] J. Hansknecht and M. Poelker, Synchronous photoinjection using a frequency-doubled gain-switched fiber-coupled seed laser and ErYb-doped fiber amplifier, Phys. Rev. Accel. Beams 9, 063501 (2006).

[7] D.-X. Wang, G. A. Krafft, and R. Abbott, Jefferson Laboratory Technical Report No. TN-94-054.

[8] M. Poelker and J. Hansknecht, in Proceedings of the Seventh International Workshop on Polarized Gas Targets and Polarized Beams, AIP Conference Proceedings 421, edited by R. J. Holt and M. A. Miller (AIP, Woodbury, NY, 1998), p. 270.

[9] Igor Pro Version 6.2, Wavemetrics Inc, Lake Oswego, OR 97035 www.wavemetrics.com.

[10] ASTRA: A space charge tracking algorithm; http://www .desy.de/ mpyflo/ and, http://www.desy.de/ mpyflo/ Astra_manual/Astra-Manual_V3.1.pdf.

[11] I. V. Bazarov, B. M. Dunham, Y. Li, X. Liu, D. G. Ouzounov, C. K. Sinclair, F. Hannon, and T. Miyajima, Thermal emittance and response time measurements of negative electron affinity photocathodes, J. Appl. Phys. 103, 054901 (2008). 